\title{
Electro-driven in situ constructing functional layer using amphoteric molecule: The role of tryptophan in ion sieving
}

Dongyu Zhang, Chenxiao Jiang*, Yuanyuan Li, Muhammad Aamir Shehzad, Xin Wang, Yaoming Wang and Tongwen $X u^{*}$

CAS Key Laboratory of Soft Matter Chemistry, Collaborative Innovation Center of Chemistry for Energy Materials, School of Chemistry and Materials Science, University of Science2 and Technology of China, Hefei, Anhui 230026, People's Republic of China

*Corresponding author: Chenxiao Jiang: jcx11@ustc.edu.cn; TongwenXu: twxu@ustc.edu.cn;
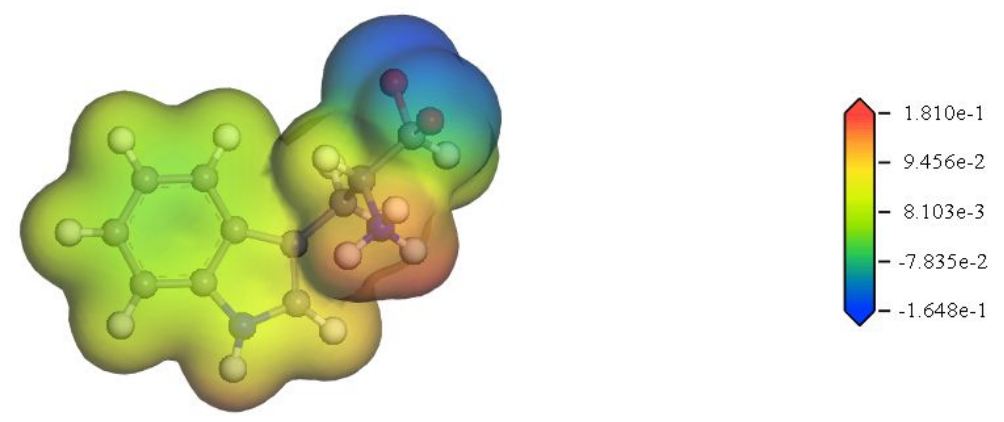

Figure S1. Charge density distribution of DL-tryptophan at pH 5.89(isoelectric point).
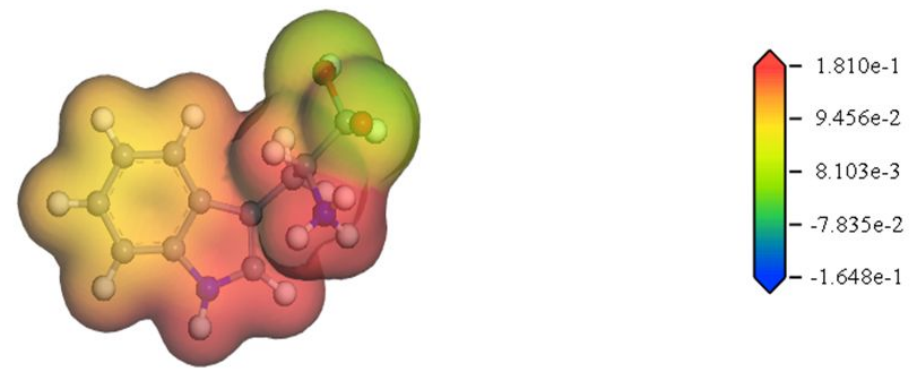
Figure S2. Charge density distribution of DL-tryptophan at $\mathrm{pH} 1$.
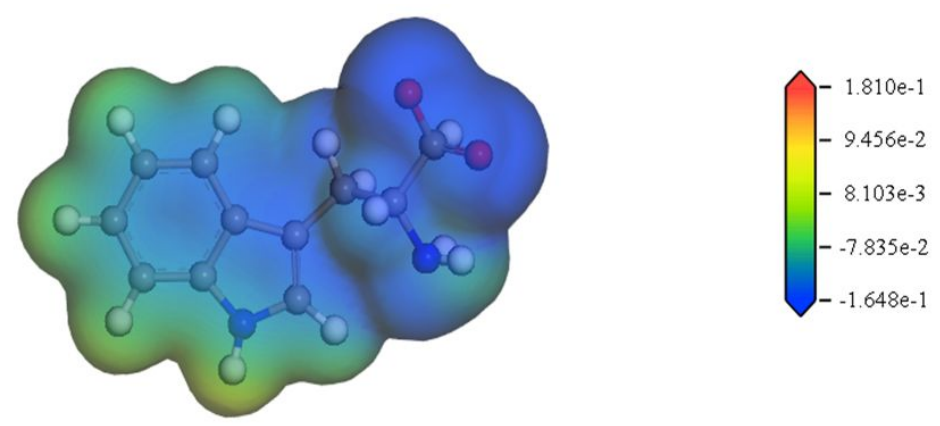

Figure S3. Charge density distribution of DL-tryptophan at pH 11.

\section{DFT Calculations.}

The charge density distributions as shown in Figure S1-S3 were calculated using an allelectron method within the Perdew-Wang 91 (PW91) form of generalized gradient approximation $(\mathrm{GGA})^{1-2}$ for the exchange-correlation term, as implemented in the $\mathrm{DMol}^{3}$ code..$^{3-4}$ The double precision numerical basis sets including $\mathrm{p}$ polarization (DNP) were adopted. The energy in each geometry optimization cycle was converged to within $1 \times 10^{-5}$ Hartree with a maximum displacement and force of $5 \times 10^{-3} \AA$ and $2 \times 10^{-3}$ Hartree/ $\AA$, respectively. The solvent effect of the water medium was described using the conductor-like screening model (COSMO). ${ }^{5-6} \mathrm{COSMO}$ is a continuum solvent model where the solute molecule forms a cavity within the dielectric continuum of permittivity that represents the solvent. 


\section{Current-Voltage Curves Measurements}

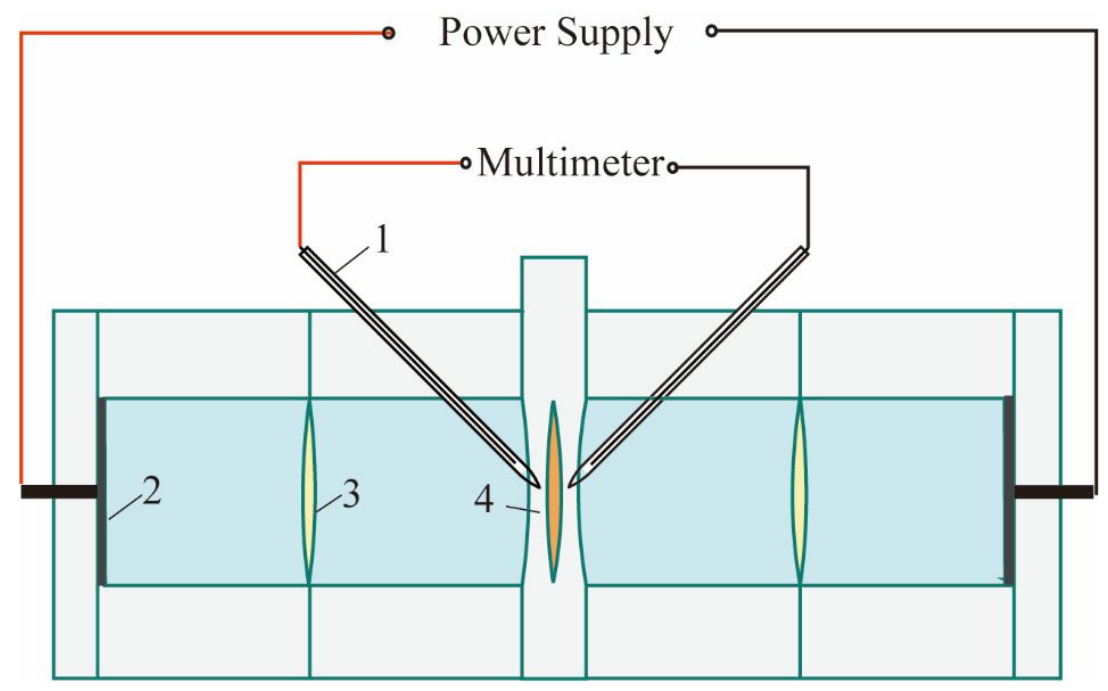

Figure S4. The experimental set up (CJ-MPMD-1) for I-V curves measurements: 1 is the reference electrode; 2 is the electrode; 3 is the supporting membrane (Nafion ${ }^{\circledR 117), ~ a n d ~} 4$ is the tested membrane.

The current-voltage curves were measured by a custom-made experimental set-up as shown in Figure S4. The set-up was powered by a direct-current power supply in constant current mode with two ruthenium-plated titanium electrodes. A multimeter $\left(\mathrm{VC} 800 \mathrm{C}^{+}\right.$, Shenzhen city station win Technology Co. Ltd., China) was connected to two Ag-AgCl reference electrodes which were located approximately $2-3 \mathrm{~mm}$ away from the tested membrane surface. The modified surface of the tested membrane was facing the anode. 


\section{Electrochemical Impedance Spectroscopy Measurements}

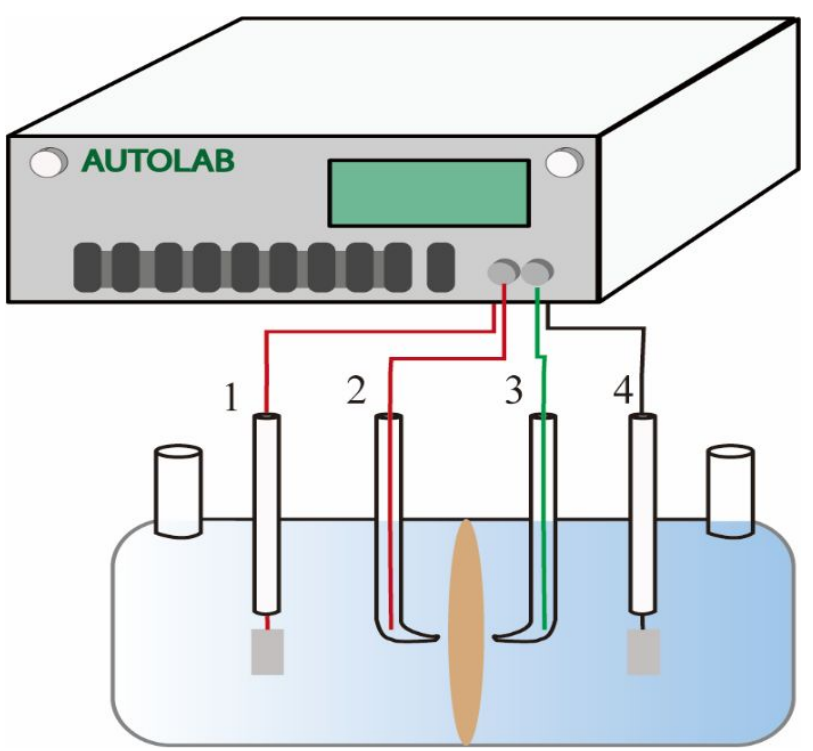

Figure S5. The experimental set-up for the EIS measurements: 1 is the working electrode; 2 is the sense electrode; 3 is the reference electrode; 4 is the counter electrode.

The electrochemical impedance spectroscopy (EIS) measurements were performed using a two-compartment glass cell as shown in Figure S5. The working and the counter electrodes were platinum electrodes. Whereas the sense and the sense electrodes were $\mathrm{Ag} / \mathrm{AgCl}$ electrodes. During the EIS test, an AC sinusoidal signal was supplied by the Autolab electrochemical work station through the working and counter electrodes. The potential drop across the tested membrane was measured by the sense and the reference electrodes which were set close to the membrane surface using the Haber-Luggin capillaries. 


\section{Ion Flux and Perm-selectivity Measurements}

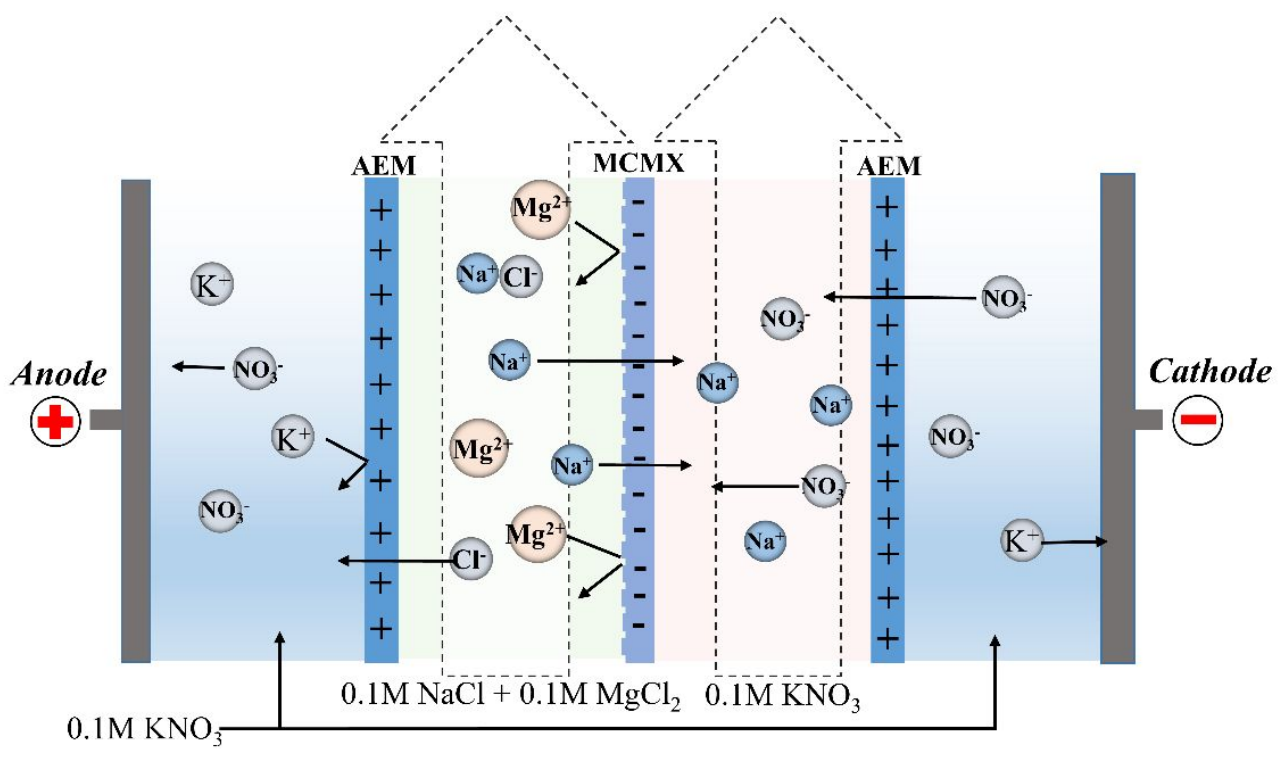

Figure S6. Schematic diagram of the electrodialysis set up (ED7-I-5) for separation test

The ion flux and perm-selectivity were measured in a custom-made electrodialysis set-up as shown in Figure S6. The tested membrane was clamped in the middle by silica gel gaskets and plates. The modified surface of the tested membrane was facing the anode, and the electrolyte solution in each compartment was circulated from the bottom up. The anode and cathode were ruthenium-plated titanium electrodes.

Table S1 Ion Flux and Perm-selectivity of Membranes

\begin{tabular}{cccc}
\hline \hline Membrane & $J_{\mathrm{Na}} \cdot 10^{-8}\left(\mathrm{~mol} \cdot \mathrm{cm}^{-2} \cdot \mathrm{S}^{-1}\right)$ & $J_{\mathrm{Mg}} \cdot 10^{-9}\left(\mathrm{~mol} \cdot \mathrm{cm}^{-2} \cdot \mathrm{S}^{-1}\right)$ & $P_{\mathrm{Mg}^{2+}}^{\mathrm{aa}^{+}}$ \\
\hline Pristine CMX & 2.06 & 14.58 & 1.41 \\
MCEM-5 & 1.51 & 2.47 & 6.12 \\
MCEM-10 & 1.58 & 0.96 & 16.36 \\
MCEM-15 & 0.97 & 0.28 & 35.13 \\
Neosepta CIMS & 4.76 & 10.58 & 4.50 \\
\hline \hline
\end{tabular}


5. XPS Analysis

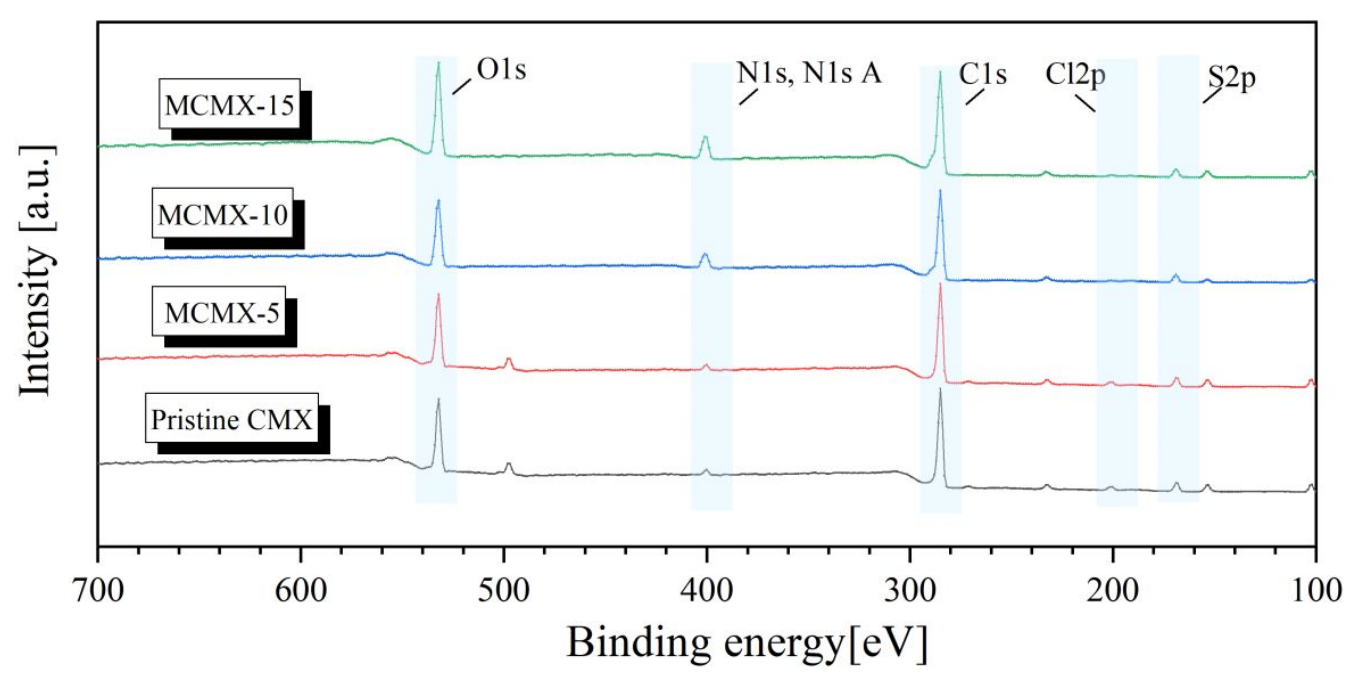

Figure S7. XPS survey spectrum of the pristine CMX surface and modified CMX surface. 


\section{References}

(1) Perdew, J. P.; Chevary, J. A.; Vosko, S. H.; Jackson, K. A.; Pederson, M. R.; Singh, D. J.;

Fiolhais, C. Atoms, Molecules, Solids, and Surfaces: Applications of the Generalized Gradient Approximation for Exchange and Correlation. Phys. Rev. B 1992, 46 (11), 6671.

(2) Perdew, J. P.; Wang, Y. Accurate and Simple Analytic Representation of the Electron-gas Correlation Energy. Phys. Rev. B 1992, 45 (23), 13244.

(3) Delley, B. An All-electron Numerical Method for Solving the Local Density Functional for Polyatomic Molecules. J. Chem. Phys. 1990, 92 (1), 508-517.

(4) Delley, B. From Molecules to Solids with the DMol 3 Approach. J. Chem. Phys. 2000, 113 (18), 7756-7764.

(5) Klamt, A.; Jonas, V.; Bürger, T.; Lohrenz, J. C. Refinement and Parametrization of COSMO-RS. J. Phys. Chem. A 1998, 102 (26), 5074-5085.

(6) Klamt, A.; Schüürmann, G. COSMO: A New Approach to Dielectric Screening in Solvents with Explicit Expressions for the Screening Energy and Its Gradient. J. Chem. Soc., Perkin Trans. 2 1993, (5), 799-805. 